\title{
Results from Gas Injection Experiment in SEPAC
}

\author{
Susumu SASAKI \\ The Institute of Space and Astronautical Science, 3-1-1 Yoshinodai, Sagamihara, \\ Kanagawa 229, Japan
}

(Received February 22, 1988; Accepted April 20, 1988)

\begin{abstract}
Neutral gas-plasma interaction in space was studied in the SEPAC experiment on the Space Shuttle, STS- 9 mission. Nitrogen gas of $10^{23}$ molecules and argon gas of $10^{21}$ molecules were repeatedly released from the orbiter at the height of $245 \mathrm{~km}$ into the ionosphere. During the gas release, an enhancement of ambient plasma density and a fluctuation of orbital potential were detected. When the gas was injected together with a high speed plasma, a CVI-like phenomenon was observed. This paper summarizes the results of the gas-plasma interaction observed in the SEPAC experiment and gives a possible explanation of the observation.
\end{abstract}

\section{Introduction}

The interaction of neutral gas with space medium (plasma, neutral gas) is a fundamental area of research in space plasma physics. The interest in this area exists also from the applicative point of view. The behavior of neutral gas in space has been studied in the large-scale chemical release experiments, usually from ground observations. On the other hand, since the Space Shuttle came into operation, the small-scale modification of space plasma by the shuttle-generated gas has been studied by in-situ measurements. Although the modification is localized around the spacecraft, the in-situ measurements by the spacecraft have revealed many physical processes involved in the gas-plasma interaction. The generation of plasma and modification of electromagnetic environments during orbiter thruster firings (PICKETT et al., 1985) are the examples of significant findings in the research.

The Space Experiment with Particle Accelerators (SEPAC) was carried out by Spacelab-1 mission in 1983 (OBAYASHI et al., 1984). It was intended to perform active experiments using a high power electron beam and a magneto-plasmadynamic arcjet (MPD-AJ). In the experiment, nitrogen and argon gas were injected into space. The well-defined gas species and the injection in a controlled manner enabled us to study the gas-plasma interaction more systematically than the previous works for the thruster gas. The interaction was observed by an on-board diagnostic package consisting of a vacuum gauge, Langmuir probe, floating probes, wave receivers, energetic electron energy analyzers, and a photometer. 


\section{Gas Injection Experiment}

Four types of gas release were studied in the SEPAC experiment; nitrogen gas injection, argon gas injection with a high speed plasma, argon gas release, and orbiter thruster firings. Figure 1 shows the location of these gas sources on the orbiter. The nitrogen and argon gas were injected from the SEPAC instruments on the pallet and the thruster gas mainly composed of $\mathrm{H}_{2} \mathrm{O}, \mathrm{N}_{2}, \mathrm{CO}$ and $\mathrm{H}_{2}$ was released from the orbiter reaction control system. Figure 2 shows the configuration of SEPAC instrumentation on the SL-1 pallet. Nitrogen gas was injected straight upward from a gas injector which was assembled in the MPD-AJ system. About $10^{23}$ molecules were released during $100 \mathrm{msec}$. Argon gas of $10^{21}$ molecules was released upward together with a high speed argon plasma from the discharge head of MPD-AJ. Argon gas of $10^{22}$ molecules was released also from the MPD casing intermittently. The modification of the plasma environment by the gas injection was studied by a diagnostic package (DGP) just close to the MPD-AJ system.

\section{Results}

\subsection{Nitrogen gas injection experiment}

The pressure measured before the gas injection was usually $(1.0-1.2) \times 10^{-6}$ Torr at the diagnostic package on the pallet. When the nitrogen gas was injected, the pressure increased to $(2-3) \times 10^{-6}$ Torr. As is shown in Fig. 3, the current of the Langmuir probe at the diagnostic package was greatly enhanced by the gas injection. The enhancement was observed only when the gas was injected into ram or near ram. At the initial phase of data analysis, this effect was believed to be related to a kind of gas ionization due to a gas-plasma interaction (SASAKI et al., 1984). However, later analysis showed that this effect could be explained by the gas-plasma collisional interaction near the orbiter (SASAKI et al., 1985). The ambient plasma drifting with respect to the orbiter at the orbital speed $(7.5 \mathrm{~km} / \mathrm{sec})$ was scattered by the gas molecules and reached the probe which was located in the local wake. There are three possible processes for the collisional interaction, viz., elastic collision, ion-atom interchange and charge exchange. The cross sectional area for the collisional interaction estimated from the plasma density at the probe is around $2 \times 10^{-21} \mathrm{~m}^{2}$, which is much larger than that for the charge exchange process but is comparable to that for the other two processes. The collisional interaction produces a plasma plume moving with the orbiter. As is shown in Fig. 4, VLF waves generally accompany the enhancement of the plasma density. The generation of the waves cannot be directly explained by the gas-plasma collisional interaction but suggests that a wave-particle interaction was also involved in this process. The mechanism for the wave generation has not been identified yet, but an electric field induced in the plasma plume moving with the orbiter in the geomagnetic field is a possible energy source for the wave generation (PICKETT et al., 1985). 


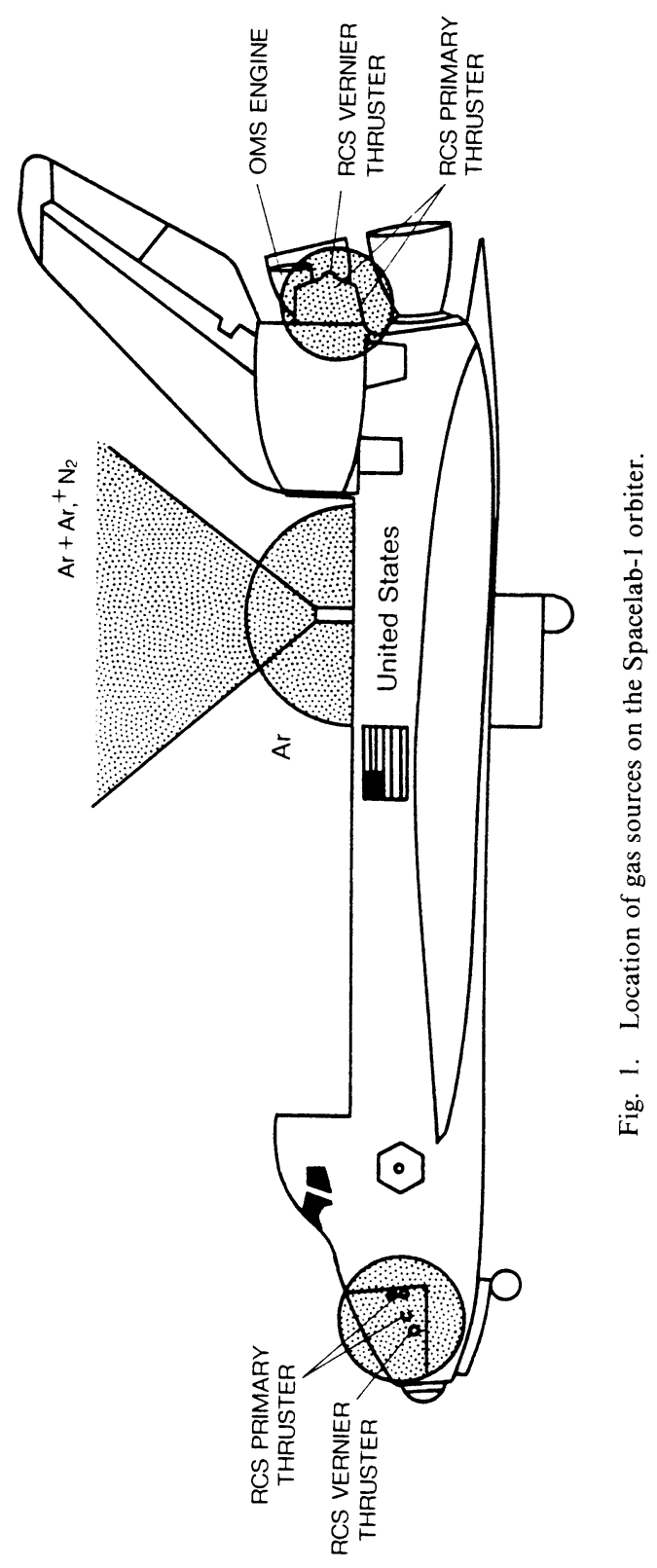




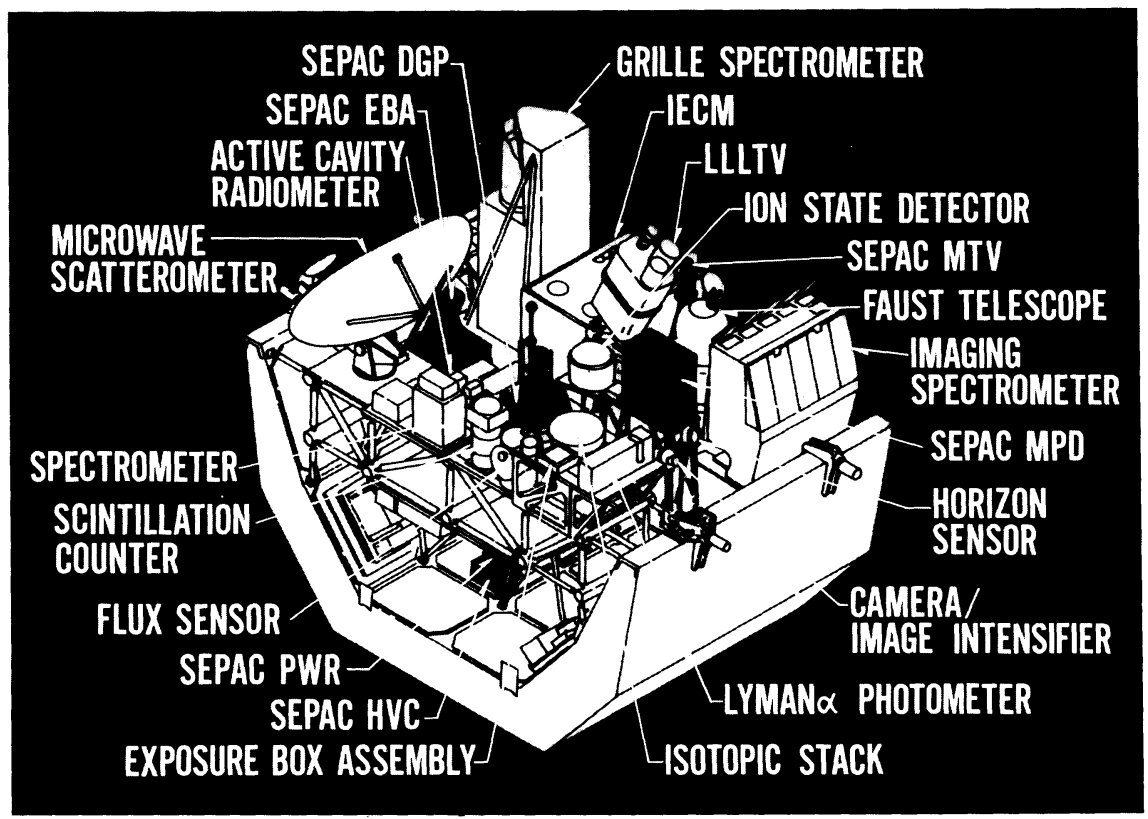

Fig. 2. Configuration of Spacelab-1 pallet instruments. SEPAC system is composed of an electron beam accelerator (EBA), a magneto-plasma-dynamic arcjet (MPD-AJ), a diagnostic package (DGP), a monitor TV camera (MTV), and power subsystems (PWR, HVC).

\subsection{Argon gas injection experiment with a high speed plasma}

Figure 5 illustrates the operation of the magneto-plasma dynamic arcjet. First, $10^{21}$ molecules of argon gas are released in coaxial electrodes inside the discharge head. At $1 \mathrm{msec}$ later, a high voltage $(500 \mathrm{~V})$ is applied between the electrodes, generating a discharge. The produced plasma accelerated by the electromagnetic force up to $20 \mathrm{~km} / \mathrm{sec}$ goes out into space through the pre-ejected neutral gas plume. The discharge is terminated within $1 \mathrm{msec}$ and about $10^{19}$ pairs of ion and electron are produced. In the MPD-AJ operation in space, long-duration effects much longer than the discharge time were unexpectedly observed by the wave, particle and optical detectors. Figure 6 shows a typical example, in which an enhancement of the plasma density, a generation of VLF waves, a fluctuation of orbiter potential and an excitation of light emission were detected for several tens of milliseconds after the termination of the MPD-AJ discharge. The observation suggests that a discharge near the orbiter was ignited for a while triggered by the plasma injection. Figure 7 shows a wave spectrum in the VLF range. The frequency of the waves is peaked near the lower hybrid frequencies of possible surrounding ions. The solid black circles in Fig. 8 show the parameter region (orbital velocity perpendicular to the magnetic field and density of ambient plasma) where the long-duration effect was observed. This effect was observed when the orbital velocity perpendicular to the magnetic 


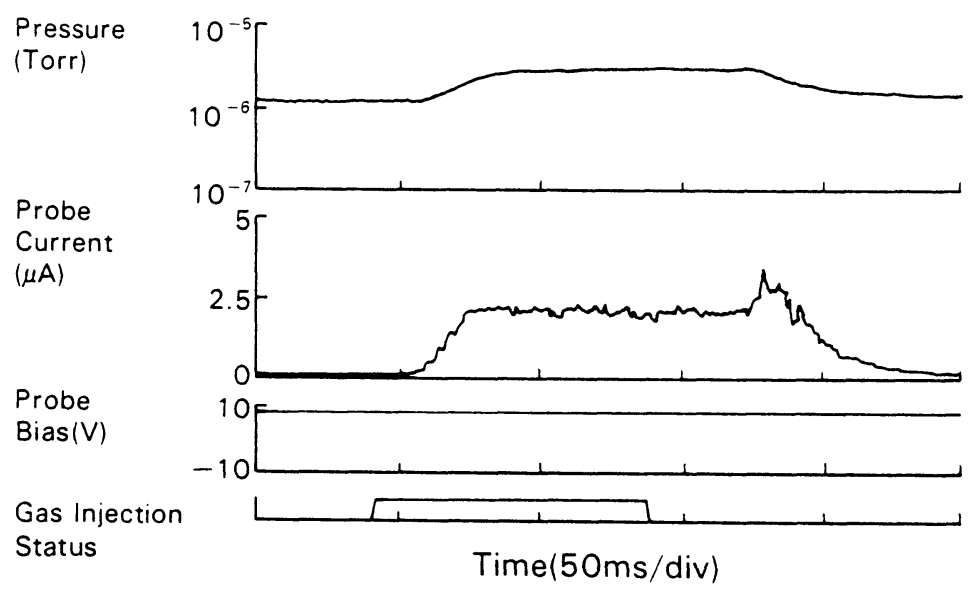

(a)

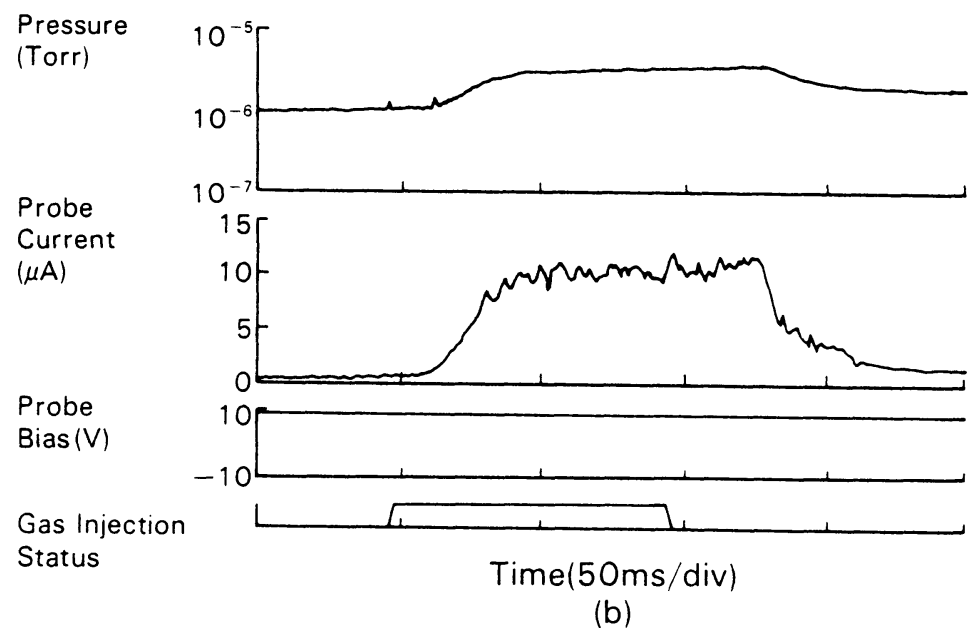

Fig. 3. Enhancement of Langmuir probe current during the nitrogen gas injection. Probe voltage is fixed at +9 V. (a) FO-4, (b) FO-9A-1.

field was relatively large and the ambient plasma density was relatively high, although these were not sufficient conditions for the effect. These features suggest that this effect is related to the concept of critical velocity ionization for the gas drifting with the orbiter (SASAKI et al., 1986). The orbital velocity $(7.5 \mathrm{~km} / \mathrm{sec}$ ) was smaller than the usual critical ionization velocity $(12.7 \mathrm{~km} / \mathrm{sec}$ for argon, 10.6 $\mathrm{km} / \mathrm{sec}$ for nitrogen and $15.6 \mathrm{~km} / \mathrm{sec}$ for oxygen) calculated based on the quasilinear theory. However, PAPADOPOULOS (1984) has proposed that specular reflection of space plasma by the orbiter surface causes counterstreaming with respect to 


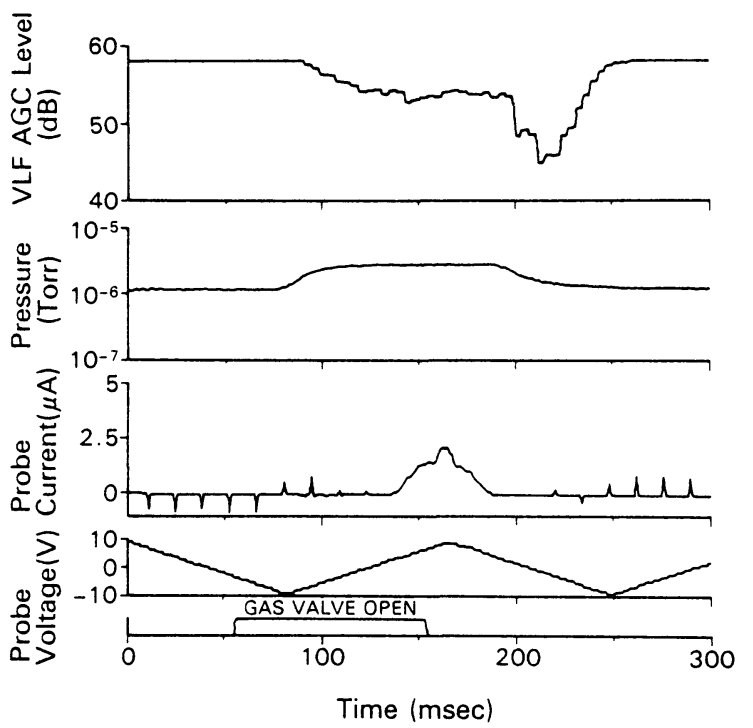

Fig. 4. Enhancement of Langmuir probe current during the nitrogen gas injection. Probe voltage is swept from $-9 \mathrm{~V}$ to $+9 \mathrm{~V}$ in $84 \mathrm{msec}$. The wave intensity integrated up to $10 \mathrm{kHz}$ is also enhanced by the gas injection.

the incoming space plasma, resulting in a relative velocity at twice the orbital velocity. He has argued in relation to the origin of the shuttle glow that the counterstreaming plasma exceeding the critical velocity will excite lower hybrid waves which cause electron heating and rapid ionization just as in the usual CVI phenomena.

\subsection{Argon gas release}

$10^{22}$ molecules of argon gas were released very slowly from the MPD casing. This was not an intensional experiment but was performed to evacuate the gas reservoir every $2.5 \mathrm{~min}$ during the MPD-AJ operation. Figure 9 shows the signal of floating probes on the DGP deck. There were three floating probes (top, middle and bottom). During the gas release, fluctuations of the floating potential to a positive side were detected by the top probe (note the high level pulses beyond $10 \mathrm{~V}$ were caused by electron beam emission and are not related to the present effect). This effect indicates that there generated an electric field between the probe and orbiter. The same effect was also observed in the argon gas injection experiment together with a high speed plasma (Fig. 6), which is possibly related to the polarization effect of the plasma moving with the orbiter (KATZ et al., 1984).

\subsection{Thruster firings}

The orbiter reaction control system consists of 38 primary thrusters and 6 

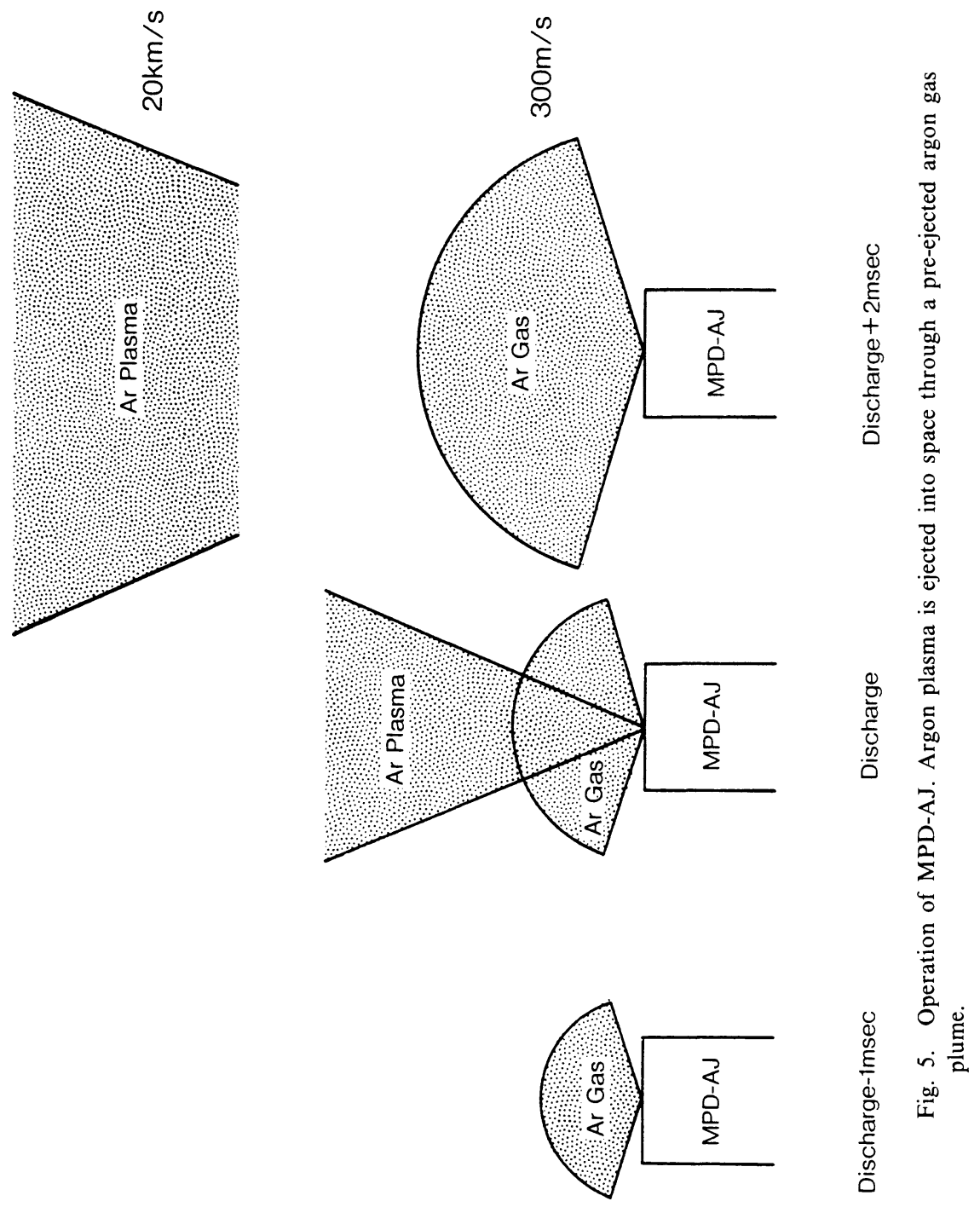


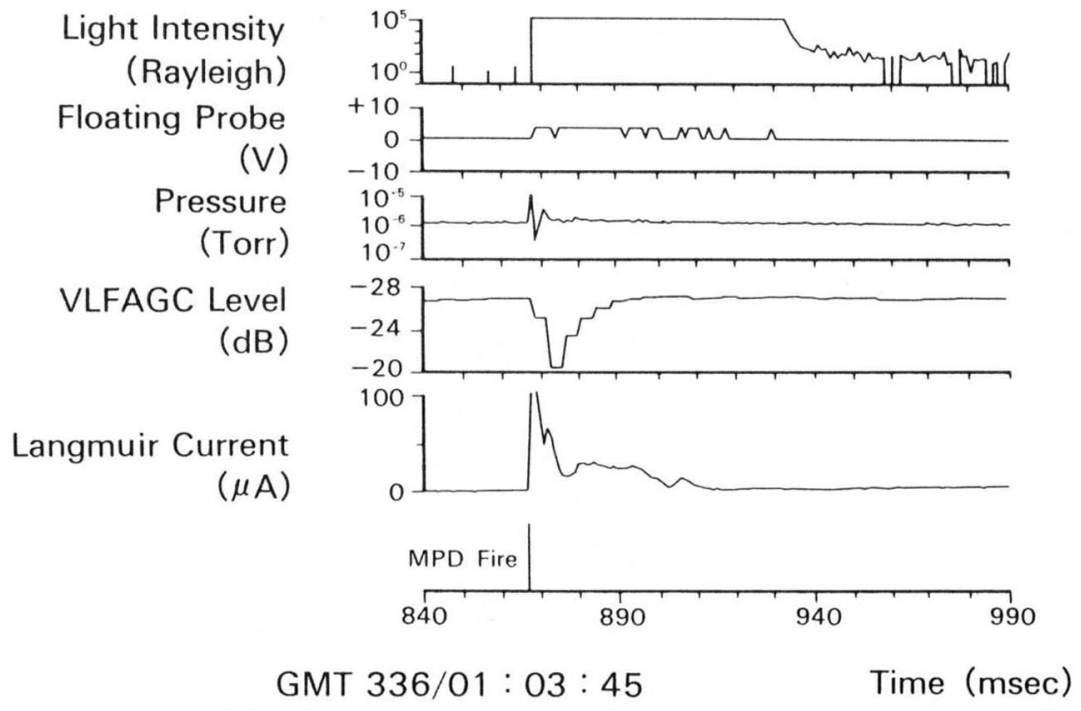

Fig. 6. Long-duration effect of diagnostic signals triggered by the MPD-AJ operation.

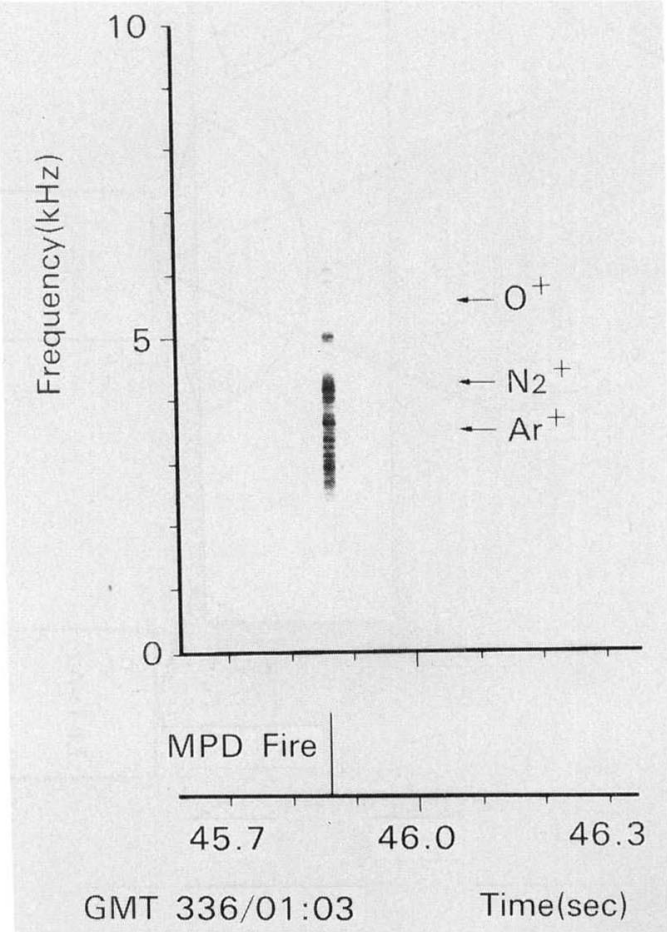

Fig. 7. Spectrum of VLF wave emission excited by the argon gas injection with a high speed plasma. The lower hybrid frequencies for oxygen, nitrogen and argon are also shown. 


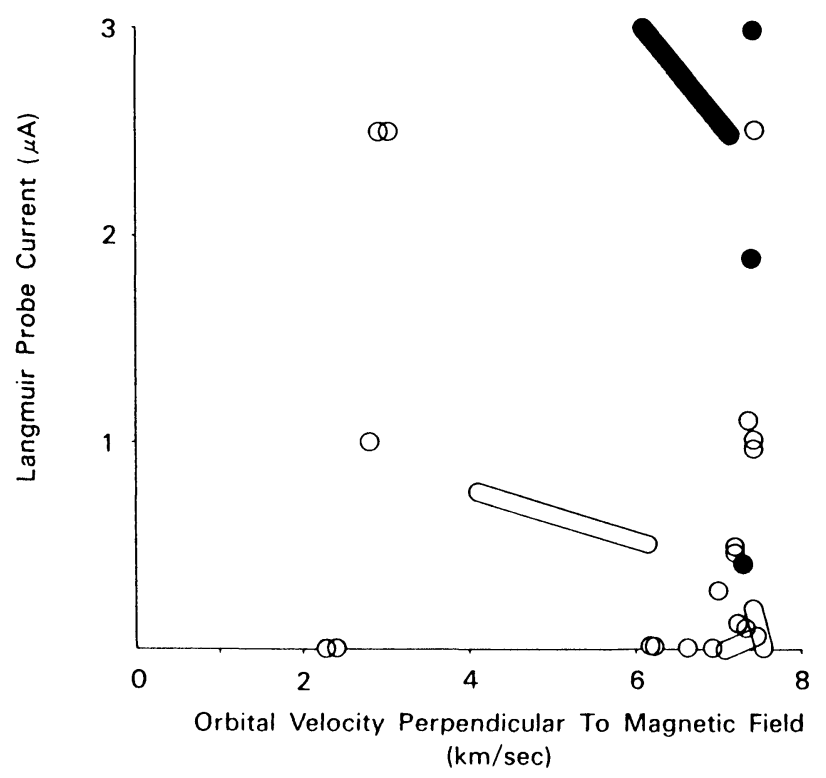

Fig. 8. Parameter region where the CVI-like phenomena are observed. Long-duration gas ionization is observed at the parameters depicted by solid black circles.

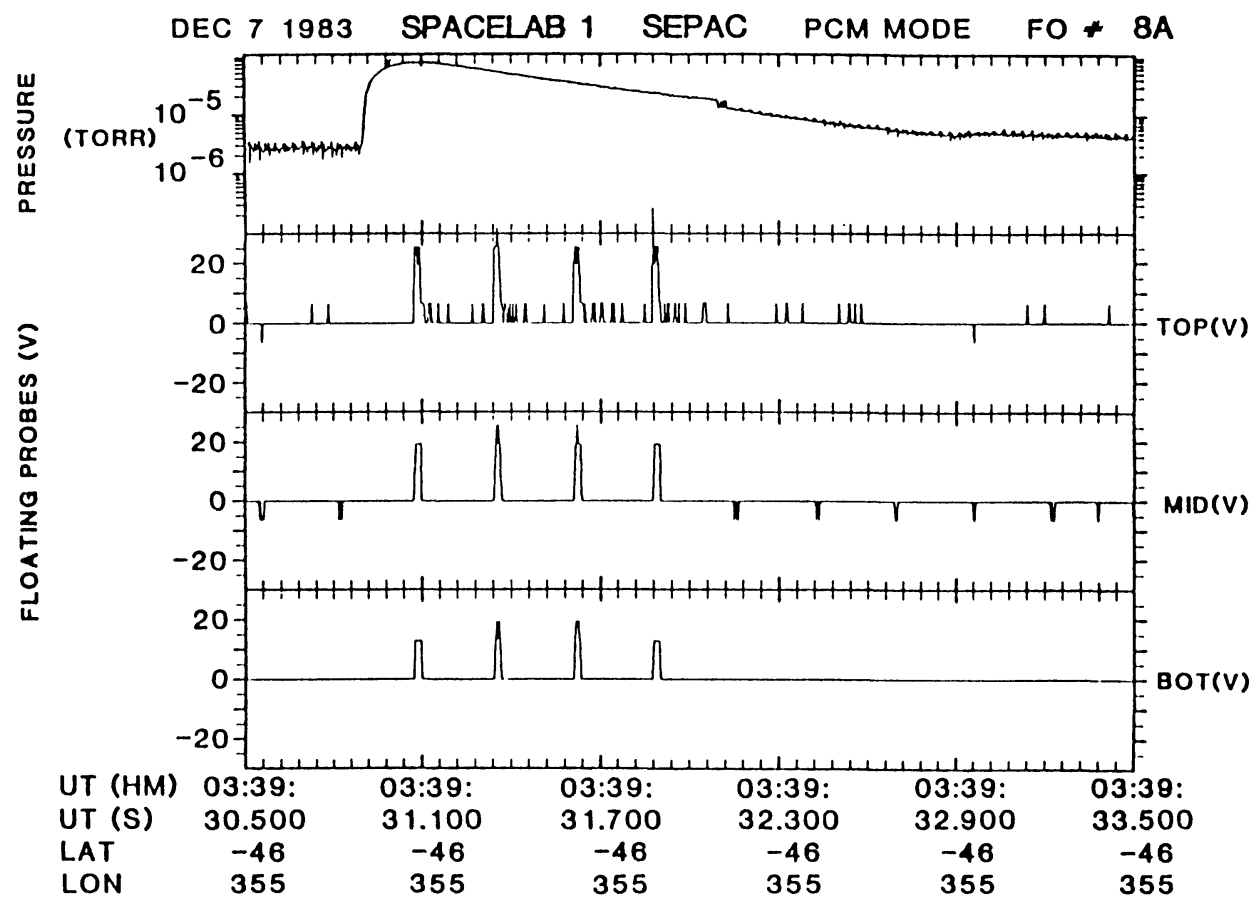

Fig. 9. Fluctuation of orbital potential during argon gas release (provided by Dr. Jill A. Marshall). 
vernier thrusters. They are located in the front and rear of the orbiter. The total number of neutrals ejected is typically $1.3 \times 10^{25}$ (based on 2 firings ejecting $163 \mathrm{~g}$ over $2 \mathrm{~s}$ ) for the vernier thruster and $9.2 \times 10^{24}$ (based on 1 firing ejecting $114 \mathrm{~g}$ over $80 \mathrm{~ms}$ ) for the primary thruster (PICKETT et al., 1985). Figure 10 shows a typical example of the diagnostic signals during the thruster firing. When the thruster was fired, a light emission and a VLF wave emission were observed. These indicate that the electromagnetic environment surrounding the orbiter is strongly modified by the thruster firing. In this particular case in which the payload bay directed toward ram, the ambient plasma density decreased during the thruster firing in contrast with the nitrogen gas injection experiment (Fig. 3). The high-density gas cloud generated by the thruster firing prevented the ambient plasma from entering the pallet. The density gradient of the plasma behind the gas cloud may be a cause for the VLF wave generation (STONE and SAMIR, 1986). In a case in which the payload bay was in wake, the density of the ambient plasma was enhanced during the thruster firing and no remarkable wave emission was detected (KAWASHIMA et al., 1985).

\section{Summary}

The SEPAC experiment provided an opportunity to study various kinds of gas-plasma interaction in space. Figure 11 illustrates a summary of possible physical

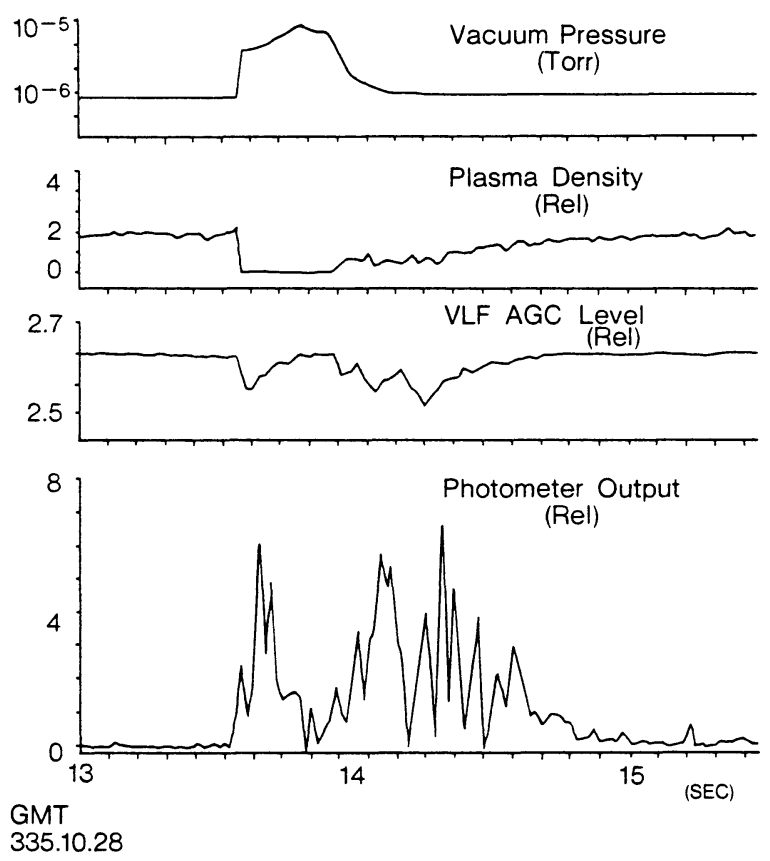

Fig. 10. Change of diagnostic signals during the thruster firing. 


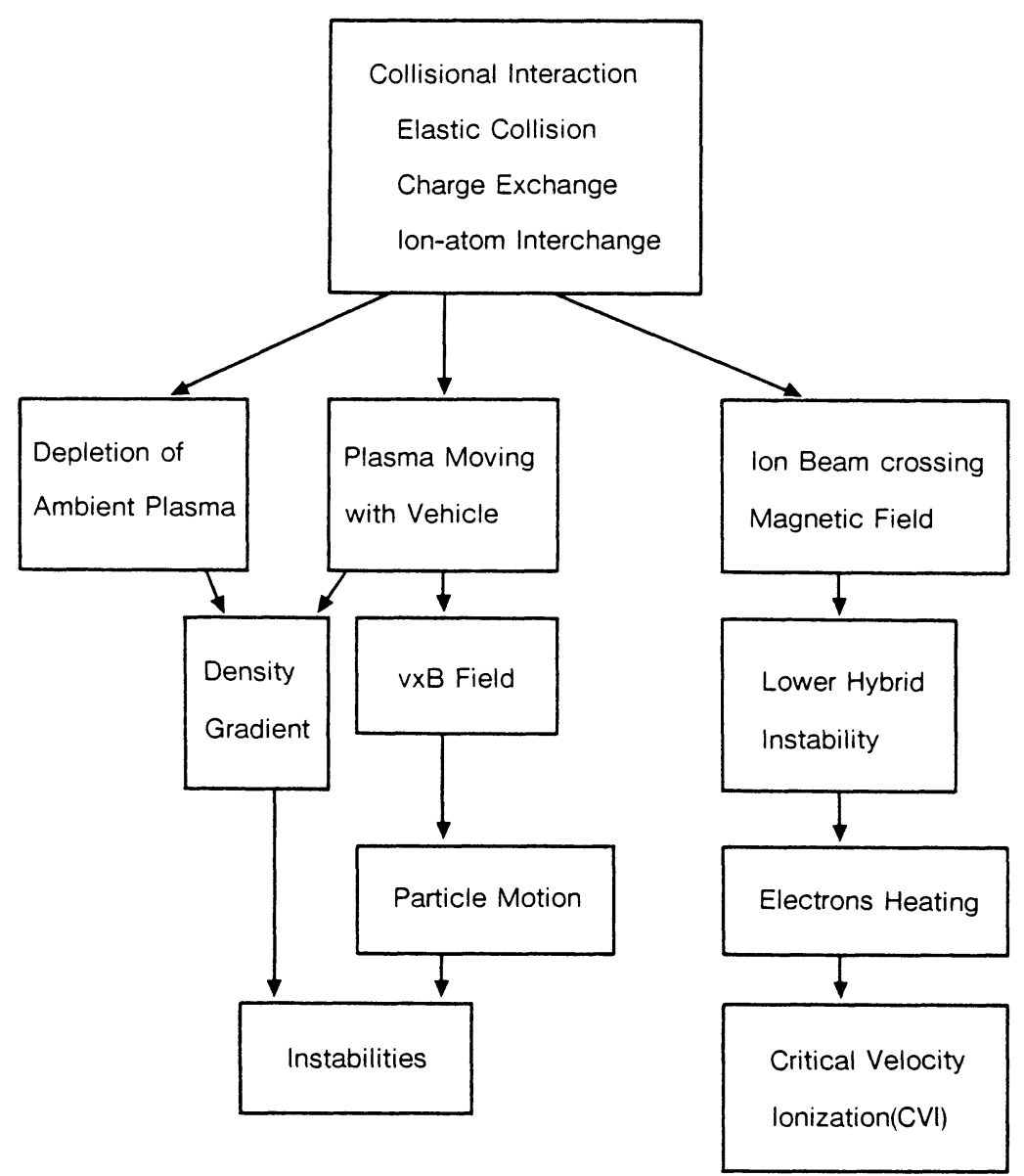

Fig. 11. Summary of possible physical processes in the gas-plasma interaction in space.

processes involved in the gas-plasma interaction. There are three major processes after the collisional interaction. Collisional interaction with a high-density gas cloud causes a depletion of plasma behind the gas cloud, generating the plasma instabilities by the density gradient. The depletion of the ambient plasma and associated wave emission were observed during the thruster firing. On the other hand, the collisional interaction produces a plasma plume moving with the orbiter, producing an $\boldsymbol{E} \times \boldsymbol{B}$ electric field in the plume, resulting in a wave particle interaction. This process was observed in nitrogen gas injection experiment under a special orbiter configuration. The collisional interaction could produce an ion beam, generating the lower hybrid instability and results in the ionization. This process is believed to be observed in the argon gas injection experiment triggered by a high speed plasma 
injection. In the SEPAC experiment, the phenomena induced by the gas release were measured at a single point on the pallet, that was insufficient to understand the total processes involved in the gas-plasma interaction in space. In order to understand the physical mechanism more definitely, multi-point measurements surrounding the spacecraft are required in the future research.

\section{REFERENCES}

Katz, I., D. E. Parks, D. L. Cookes, and J. R. Lilley, Jr., Polarization of spacecraft generated plasma clouds, Geophys. Res. Lett., 11, 1115-1116, 1984.

Kawashima, N., M. Yanagisawa, S. Sasaki, K. Kuriki, T. Obayashi, T. Neubert, P. R. Williamson, P. M. Banks, W. T. Roberts, D. L. Reasoner, W. W. L. TAylor, and J. L. Burch, Vacuum and electromagnetic environment measured in SL-1 SEPAC, ISAS Res. Note, RN-318, 1985.

Obayashi, T., N. Kawashima, K. Kuriki, M. Nagatomo, K. Ninomiya, S. Sasaki, M. Yanagisawa, M. Ejiri, W. T. Roberts, C. R. Chappell, D. L. Reasoner, J. L. Burch, W. L. Taylor, P. M. Banks, P. R. Williamson, and O. K. GarRiott, Space experiment with particle accelerators, Science, 225, 195-196, 1984.

Papadopoulos, K., On the shuttle glow (the plasma alternative), Radio Science, 19, 571-577, 1984.

Pickett, J. S., G. B. Murphy, W. S. Kurth, C. K. Goertz, and S. D. Shawhan, Effects of chemical releases by the STS 3 orbiter on the ionosphere, J. Geophys. Res., 90, 3487-3497, 1985.

Sasaki, S., N. Kawashima, K. Kuriki, M. Yanagisawa, T. Obayashi, M. Nagatomo, K. Ninomiya, M. Ejiri, I. Kudo, W. T. Roberts, C. R. Chappell, D. L. Reasoner, J. L. Burch, W. L. Taylor, P. M. BANKS, P. R. Williamson, and O. K. Garriott, Gas ionization phenomena in SEPAC SPACELAB-1 experiment, ISAS Res. Note, RN-259, 1984.

Sasaki, S., S. Kubota, N. Kawashima, K. Kuriki, M. Yanagisawa, T. Obayashi, W. T. Roberts, D. L. Reasoner, W. W. L. Taylor, P. R. Williamson, P. M. Banks, and J. L. Burch, An enhancement of plasma density by neutral gas injection observed in SEPAC Spacelab-1 experiment, J. Geomag. Geoelectr., 37, 883-894, 1985.

Sasaki, S., N. Kawashima, K. Kuriki, M. Yanagisawa, T. Obayashi, W. T. Roberts, D. L. Reasoner, W. W. L. Taylor, P. R. Williamson, P. M. Banks, and J. L. Burch, Gas ionization induced by a high speed plasma injection in space, Geophys. Res. Lett., 13, 434-437, 1986.

STONE, N. H. and U. SAMIR, The plasma dynamics of hypersonic spacecraft: Applications of laboratory simulations and active in situ experiments, in Space Technology Plasma Issues in 2001, pp. 127-148, JPL publication 86-49, 1986. 